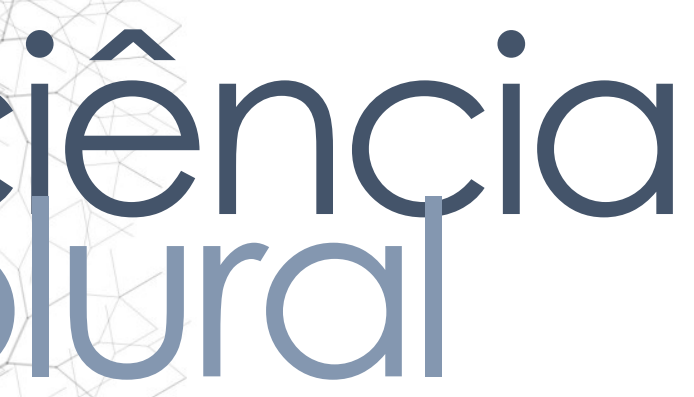

\title{
MORBIDADE E MORTALIDADE INFANTIL COM FOCO NAS CAUSAS PERINATAIS NO NORDESTE BRASILEIRO
}

Analysis of morbidity and mortality indicators of perinatal causes in children under one year in northeastern Brazilian

Analisis de los indicadores de morbilidad y mortalidad de causas perinatales en hijos bajo un año en el norte brasileño

Elionara Aline Fernandes Moreira • Acadêmica de enfermagem da Universidade Federal do Rio Grande do Norte-UFRN-Brasil • E-mail: elionaraline@gmail.com

Isabely Cardoso de Oliveira • Acadêmica de enfermagem da Universidade Federal do Rio Grande do Norte-UFRN-Brasil • E-mail: cardosobelly24@gmail.com

Fábia Barbosa de Andrade • Enfermeira • Doutora em Ciências da Saúde e Docente da Universidade Federal do Rio Grande do Norte-Natal-Brasil •

E-mail: fabiabarbosabr@gmail.com

Autora responsável pela correspondência:

Isabely Cardoso de Oliveira • E-mail: cardosobelly24@gmail.com 


\section{ciêncíncia
pural}

\section{RESUMO}

Introdução: Uma das fases da vida na qual o ser humano está mais sujeito a sofrer com o processo de morbimortalidade é a infância. Isso é ocasionada por diversos fatores, que tornam esse ciclo de vida o foco, quando se trata do estudo de mortalidade e morbidade infantil. Objetivo: analisar a morbidade hospitalar e mortalidade infantil por causas perinatais em menores de um ano no nordeste brasileiro no período de 2013 a 2017. Metodologia: Caracteriza-se como estudo do tipo ecológico, realizado a partir de dados secundários extraídos do Departamento de Informática do Sistema Único de Saúde oriundos do Sistema de Informação de Mortalidade e o Sistema de Informação Hospitalar do Sistema Único de Saúde, no período de 2013 a 2017. Resultados: Revelaram que as causas perinatais são grandes contribuintes para a mortalidade. Dos fatores que mais matam estão à septicemia bacteriana (30,7\%) e afecções maternas $(42,3 \%)$. O ano de 2013 e o estado da Bahia revelaram um maior número de óbitos. Quanto à morbidade pode-se observar os agravos afecções do período perinatal $(33,8 \%)$ e transtornos respiratórios do período perinatal $(49,7 \%)$ sendo aqueles com maior número de casos, além do ano de 2017 e o estado da Bahia como sendo os que apresentaram maior número de internações. Conclusões: As causas perinatais são um dos principais fatores responsáveis por gerar vítimas, tornando-se necessária a tomada de medidas. Deve-se melhorar a transmissão de conhecimentos a fim de prevenir as doenças e fortalecer a promoção à saúde e reduzir as taxas de morbimortalidade.

Palavras-Chave: Mortalidade infantil; Mortalidade neonatal; Enfermagem perinatal; Cuidado perinatal; Morbidade; Mortalidade perinatal.

\section{ABSTRACT}

Introduction: One of the stages of life in which the human being is most likely to suffer from the process of morbidity and mortality is childhood. This is caused by several factors, which make this life cycle the focus, when it comes to the study of infant mortality and morbidity. Objective: analyze hospital morbidity and infant mortality from perinatal causes in children under one year in northeastern Brazil from 2013 to 2017. Methodology: It is characterized as an ecological study, carried out from secondary data extracted from the Informatics Department of the Unified Health System from the Mortality Information System and the Hospital Information System of the Unified Health System , from 2013 to 2017. Results: They revealed that perinatal causes are major contributors to mortality. Of the factors that kill the most are bacterial septicemia (30.7\%) and maternal disorders (42.3\%). 2013 and the state of Bahia revealed a greater number of deaths. Morbidity can be observed the diseases affecting the perinatal period $(33.8 \%)$ and perinatal respiratory disorders $(49.7 \%)$ being those with the highest number of cases, in addition to 2017 and the state of Bahia as the ones with the highest number of hospitalizations. Conclusions: Perinatal causes are one of main factors responsible for generating victims, making it necessary to take action. owledge transmission should be improved in order to prevent diseases and engthen health promotion and reduce morbidity and mortality rates.

ywords: Infant mortality; Neonatal mortality; Perinatal nursing; Perinatal care;

bidity; Perinatal mortality. 


\section{ciêncíncia
pural}

\section{RESUMEN}

Introducción: Una de las etapas de la vida en la que el ser humano tiene más probabilidades de sufrir el proceso de morbilidad y mortalidad es la infancia. Esto es causado por varios factores, que hacen que este ciclo de vida sea el foco, cuando se trata del estudio de la mortalidad y la morbilidad infantil. Objetivo: analizar la morbilidad hospitalaria y la mortalidad infantil por causas perinatales en niños menores de un año en el noreste de Brasil de 2013 a 2017. Metodología: Se caracteriza por ser un estudio ecológico, realizado a partir de datos secundarios extraídos del Departamento de Informática del Sistema Unificado de Salud del Sistema de Información de Mortalidad y del Sistema de Información Hospitalaria del Sistema Unificado de Salud, de 2013 a 2017. Resultados: Revelaron que las causas perinatales son los principales contribuyentes a la mortalidad. De los factores que más matan son la septicemia bacteriana (30,7\%) y los trastornos maternos (42,3\%). 2013 y el estado de Bahía revelaron un mayor número de muertes. Se pueden observar la morbilidad de las enfermedades que afectan al período perinatal $(33,8 \%)$ y trastornos respiratorios perinatales $(49,7 \%)$ siendo aquellos con mayor número de casos, además de 2017 y el estado de Bahía como los que tienen el mayor número de hospitalizaciones. Conclusiones: Las causas perinatales son uno de los principales factores responsables de generar víctimas, por lo que es necesario tomar medidas. Debe mejorarse la transmisión de los conocimientos a fin de prevenir enfermedades y fortalecer la promoción de la salud y reducir las tasas de morbilidad y mortalidad.

Palabras clave: Mortalidad infantil; Mortalidad neonatal; Enfermería perinatal;

Cuidado perinatal; Morbilidad; Mortalidad perinatal. 


\section{Introdução}

O processo de morbimortalidade na infância possui causas multifatoriais, por fatores internos e externos. Os órgãos ainda estão em processo de maturação final, o sistema imunológico ainda é imaturo, sem falar em agravantes como a situação socioeconômica do indivíduo, a prematuridade, más formações congênitas e baixo peso ao nascer ${ }^{1}$.

A mortalidade infantil trata-se de mortes em menores de um ano, e compreende óbitos neonatais e pós-neonatais. As mortes neonatais ocorrem em crianças com até 27 dias de vida, geralmente por causas biológicas como malformações genéticas ou em razão das questões referentes à gestação e complicação no parto, e pós neonatais que correspondem as mortes do $28^{\circ}$ ao $364^{\circ}$ dia de vida, com influências externas que podem estar associadas às desigualdades sociais, condições de moradia e situação socioeconômica que implica no surgimento de doenças que de modo geral são evitáveis ${ }^{2}$.

Relacionado às causas de mortalidade, $\mathrm{Liu}^{3}$ versam que no mundo, no intervalo de 2000 a 2013, ocorreram 6.3 milhões de mortes em crianças, sendo que 3.257 milhões $(51,8 \%)$ morreram devidas causas infecciosas, sendo as principais: pneumonia $(14,9 \%)$, diarreia $(9,2 \%)$ e malária $(7,3 \%)$. No Chile, em uma amostra de 9.527 mortes em lactentes, um terço delas foi causada por motivos relacionados à prematuridade (2.856) e devido à síndrome da morte súbita infantil (319) .

Já as morbidades hospitalares, nos Estados Unidos destacam-se que de 2,1\% a 10,7\% das crianças que tem asma apresentaram casos de hospitalização ${ }^{5}$. No Brasil, em pesquisa realizada em hospitais mostra que a rede pública recebeu no ano de 2013 cerca de 190.000 pacientes para a internação, com uma taxa de 331 internações a cada 100.000 habitantes, apresentando na Região Sul as taxas mais elevadas e no Norte as mais baixas; ressalta-se ainda que as taxas para crianças menores de um ano foram de mais de 1.000 internações por 100.000 habitantes $^{6}$.

A pesquisa apresentou como foco a região nordeste, tendo em vista que essa ião está entre as que apresentam as maiores taxas de mortalidade e morbidade do sil, de modo que faz-se necessário avaliar a realidade sociodemográfica e 
epidemiológica, bem como pela importante atuação assistencial nos diferentes níveis do cuidado. Assim, esta pesquisa se propôs analisar a morbidade hospitalar e mortalidade infantil por causas perinatais em menores de um ano no nordeste brasileiro no período de 2013 a 2017.

\section{Metodologia}

Caracteriza-se como estudo do tipo ecológico, realizado a partir de dados secundários extraídos do Departamento de Informática do Sistema Único de Saúde (DATASUS) oriundos do Sistema de Informação de Mortalidade (SIM) e o Sistema de Informação Hospitalar do SUS (SIH-SUS), no período de 2013 a 2017.

Para Bando $^{7}$ o estudo ecológico possui algumas limitações, pois ao usá-lo, as associações que são encontradas em uma população, possivelmente não poderão ser aplicadas a nível individual. Por outro lado, é um tipo de estudo relevante, uma vez que ele gera abertura para um caminho de novas pesquisas e hipóteses, sem contar que é bastante útil quando as intervenções ocorrem na área do agregado populacional.

A escolha da série histórica estudada se deu por tratar dos últimos cinco anos disponíveis completos do SIM e SIH-SUS no momento da extração dos dados e completude do banco de dados para esta pesquisa, em meados de agosto e setembro de 2019. O motivo da escolha se deu por ser uma região extensa e com características sociodemográficas semelhantes.

O Brasil tem uma área total de 8.515.767,049, desse território a região nordeste ocupa um espaço de 1.554.291,6 km², sendo 18,2\% de todo o território do Brasil. Possui uma população de 57.071.654 milhões de habitantes, segundo a estimativa feita pelo IBGE para 2019, uma densidade demográfica de 36,49 hab./ km² e 1794 municípios, além de 9 estados, sendo eles: Alagoas (3.337.357 milhões), Bahia (14.873.064 milhões), Ceará (9.132.078 milhões), Maranhão (7.075.181 milhões), Paraíba (4.018.127 milhões), Plauí (3.273.227 milhões), Pernambuco (9.557.071 milhões), Rio Grande do Norte 3.506.853 milhões) e Sergipe (2.298.696 milhões) ${ }^{8}$. Utilizaram-se como variáveis ependentes, óbitos em menores de um ano e adoecimento hospitalar. 
Neste estudo foi usado à lista com os agravos de mortalidade e morbidade, que trazem em seu conteúdo 21 capítulos com diferentes classificações para doenças. $\mathrm{O}$ trabalho teve como foco o capítulo XVI - Algumas afecções originadas no período perinatal. O capítulo XVI da lista de mortalidade contém uma variação de agravos que é codificado do P00 até o P96, enquanto a lista de morbidade apresenta a mesma variação sendo codificado do 245 até o 253 .

Dentre os 59 agravos que correspondem à lista de mortalidade do capítulo XVI do CID-10, foram selecionados os mais prevalentes no que diz respeito aos dados de mortalidade e morbidade. Foi com base nos dados obtidos desses agravos que foram realizadas as análises e construção das tabelas e dos resultados. Os agravos foram os seguintes: Septicemia Bacteriana; Desconforto Respiratório; Gestação Curta e Baixo Peso; Outras Afecções Maternas; Asfixia Ao Nascer; Afecções do período perinatal; Transtornos respiratórios do período perinatal; Crescimento, desnutrição, gestação curta e baixo peso; Infecções do período perinatal; Doenças infecciosas e parasitárias congênitas.

A etapa da análise dos dados foi feita com o auxílio do programa Software Microsoft Excel, por meio dele os dados coletados foram organizados e tratados, foi realizada a exclusão de informações desnecessárias que se encontravam no cabeçalho e rodapé de cada arquivo, além da substituição do "_" por “0".

Ocorreu também a ordenação dos municípios através do código estabelecido pelo IBGE, intitulado CODIGOMUN, e por fim a exclusão dos municípios ignorados e a classificação deles de acordo com a ordem alfabética. Concluída estaa etapa, os dados foram inseridos no Software Statistical Package for the Social Sciences (SPSS), versão 20.0 .

Para análise dos dados foram utilizados cálculo de frequências absolutas, relativas, média, mediana, desvio padrão e de posse dos resultados foram elaborados iguras que serviram de suporte para construção dos resultados e discussão. 


\section{Resultados}

A tabela 1 apresenta os valores relativos da mortalidade infantil dentre os cinco maiores agravos analisados, na qual é possível perceber que o ano responsável pelo maior número de mortes foi o de 2013 com 4166 óbitos, já o que apresentou o menor número foi o de 2016 com 3499 mortes.

Porém o agravo que foi responsável por causar o maior número de mortes em lactentes foi o de septicemia bacteriana no ano de 2015, que foi de 1133 (30,7\%) óbitos. Em paralelo a isso, temos a doença com menor número de mortes, a asfixia ao nascer no ano de 2017, com 363 (10\%) vítimas.

Tabela 1 - Distribuição da mortalidade infantil de acordo com as causas incluídas no estudo e estratificada por ano (2013-2017) no Nordeste do Brasil. Natal-RN, 2020.

\begin{tabular}{ccccccc}
\hline \multirow{2}{*}{ Ano } & $\begin{array}{c}\text { Septicemia } \\
\text { Bacteriana }\end{array}$ & $\begin{array}{c}\text { Desconforto } \\
\text { Respiratório }\end{array}$ & $\begin{array}{c}\text { Gestação } \\
\text { Curta e } \\
\text { Baixo Peso }\end{array}$ & $\begin{array}{c}\text { Outras } \\
\text { Afecções } \\
\text { Maternas }\end{array}$ & $\begin{array}{c}\text { Asfixia Ao } \\
\text { Nascer }\end{array}$ & Total \\
\cline { 2 - 6 } & $\mathrm{n}(\%)$ & $\mathrm{n}(\%)$ & $\mathrm{n}(\%)$ & $\mathrm{n}(\%)$ & $\mathrm{n}(\%)$ & \\
\cline { 2 - 6 } 2013 & $1117(26,8 \%)$ & $1035(24,8 \%)$ & $876(21,0 \%)$ & $626(15,0 \%)$ & $512(12,2 \%)$ & 4166 \\
2014 & $1073(28,0 \%)$ & $913(23,8 \%)$ & $781(20,4 \%)$ & $569(14,8 \%)$ & $490(12,8 \%)$ & 3826 \\
2015 & $1133(30,7 \%)$ & $856(23,2 \%)$ & $646(17,5 \%)$ & $624(16,9 \%)$ & $422(11,4 \%)$ & 3681 \\
2016 & $1046(29,8 \%)$ & $772(22,0 \%)$ & $689(19,6 \%)$ & $594(16,9 \%)$ & $398(11,3 \%)$ & 3499 \\
2017 & $1077(29,9 \%)$ & $815(22,6 \%)$ & $691(19,1 \%)$ & $653(18,1 \%)$ & $363(10,0 \%)$ & 3599 \\
\hline
\end{tabular}

Fonte: DATASUS (2013-2017)

Na tabela 2, foi elaborada uma análise de mortalidade voltada aos Estados.

Percebe-se que o estado da Bahia foi o que apresentou maior número de mortes, com 4880 casos. Entretanto, o agravo que mais mata é o de outras afecções maternas, com $440(42,3 \%)$ eventos, todos ocorridos no estado do Piauí.

O Estado que mostrou o menor número de mortes foi o de Sergipe, com 848 óbitos. Apesar disso o agravo asfixia ao nascer apontou os menores valores de mortalidade ocorridos no estado do Rio Grande do Norte, apenas 49 (4,4\%) corrências. 
Tabela 2 - Distribuição da mortalidade infantil com variação dos estados (20132017), no Nordeste do Brasil. Natal-RN, 2020.

\begin{tabular}{ccccccc}
\hline \multirow{2}{*}{ Estados } & $\begin{array}{c}\text { Septicemia } \\
\text { bacteriana }\end{array}$ & $\begin{array}{c}\text { Desconforto } \\
\text { respiratório }\end{array}$ & $\begin{array}{c}\text { Gestação } \\
\text { curta e } \\
\text { baixo peso }\end{array}$ & $\begin{array}{c}\text { Outras } \\
\text { afecções } \\
\text { maternas }\end{array}$ & $\begin{array}{c}\text { Asfixia ao } \\
\text { nascer }\end{array}$ & Total \\
\cline { 2 - 6 } & $\mathrm{n}(\%)$ & $\mathrm{n}(\%)$ & $\mathrm{n}(\%)$ & $\mathrm{n}(\%)$ & $\mathrm{n}(\%)$ & \\
\hline MA & $781(30,6 \%)$ & $607(23,7 \%)$ & $388(15,2 \%)$ & $368(14,4 \%)$ & $407(15,9 \%)$ & 2551 \\
PI & $153(14,7 \%)$ & $179(17,2 \%)$ & $129(12,4 \%)$ & $440(42,3 \%)$ & $139(13,3 \%)$ & 1040 \\
CE & $912(31,0 \%)$ & $777(26,4 \%)$ & $609(20,7 \%)$ & $202(6,8 \%)$ & $441(14,9 \%)$ & 2941 \\
RN & $365(32,9 \%)$ & $291(26,2 \%)$ & $228(20,5 \%)$ & $175(15,7 \%)$ & $49(4,4 \%)$ & 1108 \\
PB & $472(39,9 \%)$ & $362(30,6 \%)$ & $137(11,6 \%)$ & $125(10,5 \%)$ & $85(7,1 \%)$ & 1181 \\
PE & $661(24,1 \%)$ & $412(15,0 \%)$ & $419(15,2 \%)$ & $968(35,3 \%)$ & $281(10,2 \%)$ & 2741 \\
AL & $543(38,3 \%)$ & $522(36,8 \%)$ & $115(8,1 \%)$ & $113(7,9 \%)$ & $123(8,6 \%)$ & 1416 \\
SE & $238(28,0 \%)$ & $133(15,6 \%)$ & $153(18,0 \%)$ & $180(21,2 \%)$ & $144(16,9 \%)$ & 848 \\
BA & $1295(26,5 \%)$ & $1091(22,3 \%)$ & $1490(30,5 \%)$ & $492(10,0 \%)$ & $512(10,4 \%)$ & 4880 \\
\hline
\end{tabular}

Fonte: DATASUS (2013-2017)

$\mathrm{Na}$ tabela 3 foi realizada uma avaliação anual também com referência a morbidade. É perceptível que o ano de 2017 foi o que apresentou maior número de internações (66892). Vale ressaltar ainda que o agravo que apresentou maior destaque foi o de afecções do período perinatal no ano de 2017, com 22662 internações (33,8\%).

Em oposição a isso temos o ano de 2013 como sendo o que mostrou o menor número de internações, apenas 55297, e nesse mesmo ano o agravo de doenças infecciosas e parasitárias congênitas foi o que revelou os menores números, logo concluímos que as crianças adoecem menos por esse motivo, apenas $2344(4,2 \%)$ das internações ocorrem por esse fator.

Tabela 3 - Distribuição da morbidade infantil com variação anual (2013-2017), no Nordeste do Brasil. Natal-RN, 2020.

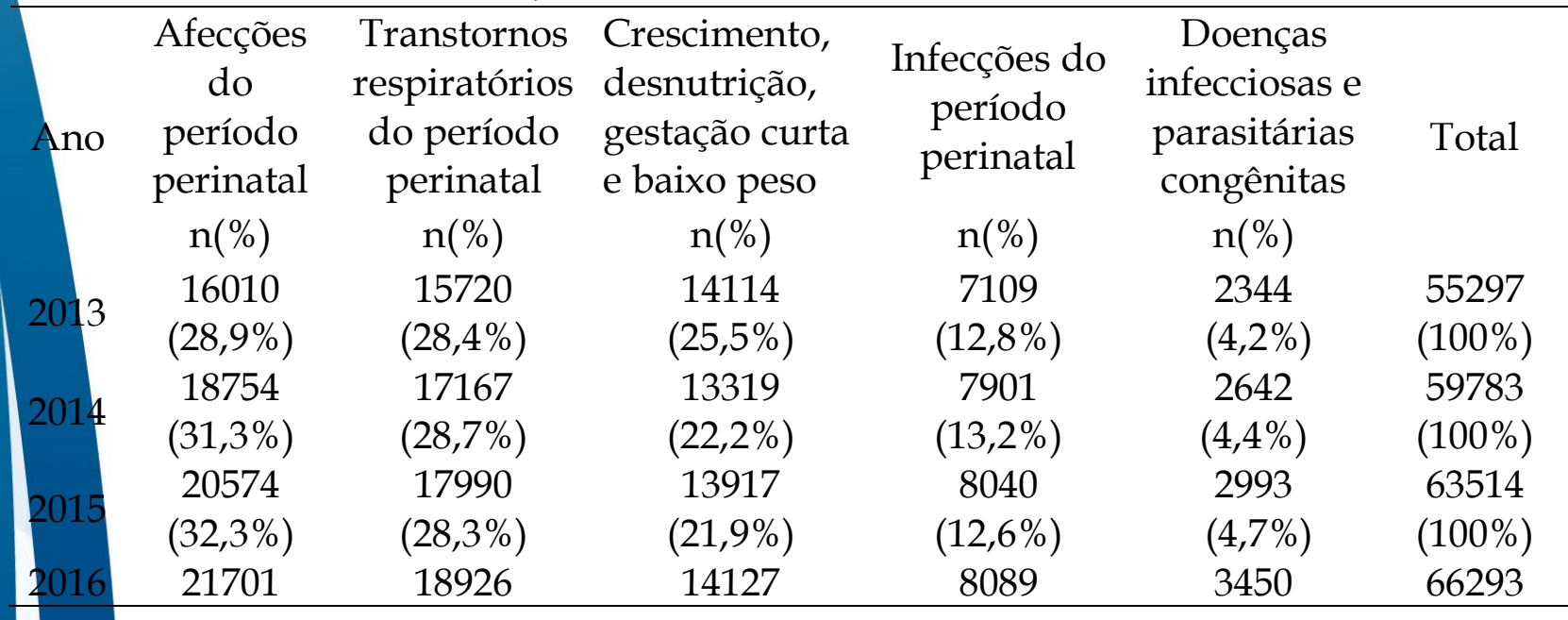




\begin{tabular}{ccccccc} 
& $(32,7 \%)$ & $(28,5 \%)$ & $(21,3 \%)$ & $(12,2 \%)$ & $(5,2 \%)$ & $(100 \%)$ \\
2017 & 22662 & 19011 & 13991 & 7949 & 3279 & 66892 \\
& $(33,8 \%)$ & $(28,4 \%)$ & $(20,9 \%)$ & $(11,8 \%)$ & $(4,9 \%)$ & $(100 \%)$ \\
\hline
\end{tabular}

Fonte: DATASUS (2013-2017)

$\mathrm{Na}$ tabela 4, quando observados os dados de morbidade de cada estado, a Bahia novamente aparece com os maiores números, foram 69350 internações. No entanto, o estado do Piauí foi o responsável pelo agravo mais prevalente, sendo os transtornos respiratórios do período perinatal, com cerca de 6436 (49,7\%) internações.

O Piauí também aparece mais uma vez dentre os que apresentaram os menores valores, sendo 12927 internações nesse estado. Já as doenças infecciosas e parasitárias congênitas são responsáveis por ser o agravo que menos gerou índices de internações hospitalares, sendo apenas $193(1,4 \%)$ eventos por esse motivo.

Tabela 4 - Distribuição da morbidade infantil com variação dos estados (2013-2017), no Nordeste do Brasil. Natal-RN, 2020.

\begin{tabular}{ccccccc} 
Estados & $\begin{array}{c}\text { Afecções do } \\
\text { período } \\
\text { perinatal }\end{array}$ & $\begin{array}{c}\text { Transtornos } \\
\text { respiratórios } \\
\text { do período } \\
\text { perinatal } \\
\mathrm{n}(\%)\end{array}$ & $\begin{array}{c}\text { Crescimento, } \\
\text { desnutrição, } \\
\text { gestação curta } \\
\text { e baixo peso } \\
\mathrm{n}(\%)\end{array}$ & $\begin{array}{c}\text { Infecções do } \\
\text { período } \\
\text { perinatal }\end{array}$ & $\begin{array}{c}\text { Doenças } \\
\text { infecciosas e } \\
\text { parasitárias } \\
\text { congênitas } \\
\mathrm{n}(\%)\end{array}$ & Total \\
MA & $4032(14,5 \%)$ & $8403(30,4 \%)$ & $7983(28,8 \%)$ & $5459(19,7 \%)$ & $1747(6,3 \%)$ & 27624 \\
PI & $3134(24,2 \%)$ & $6436(49,7 \%)$ & $1791(13,8 \%)$ & $1373(10,6 \%)$ & $193(1,4 \%)$ & 12927 \\
CE & $24169(37,9 \%)$ & $19892(31,1 \%)$ & $11832(18,5 \%)$ & $5750(9,0 \%)$ & $2118(3,3 \%)$ & 63761 \\
RN & $4806(31,1 \%)$ & $5408(35,0 \%)$ & $3917(25,3 \%)$ & $837(5,4 \%)$ & $473(3,0 \%)$ & 15441 \\
PB & $5702(37,1 \%)$ & $3651(23,7 \%)$ & $3566(23,2 \%)$ & $2007(13,0 \%)$ & $441(2,8 \%)$ & 15367 \\
PE & $27913(40,5 \%)$ & $13338(19,3 \%)$ & $15626(22,6 \%)$ & $8472(12,3 \%)$ & $3505(5,0 \%)$ & 68854 \\
AL & $5884(23,6 \%)$ & $10553(42,3 \%)$ & $4436(17,8 \%)$ & $1754(7,0 \%)$ & $2290(9,1 \%)$ & 24917 \\
SE & $3063(22,6 \%)$ & $5422(40,0 \%)$ & $2267(16,7 \%)$ & $2372(17,5 \%)$ & $414(3,0 \%)$ & 13538 \\
BA & $20998(30,2 \%)$ & $15711(22,6 \%)$ & $18050(26,0 \%)$ & $11064(15,9 \%)$ & $3527(5,0 \%)$ & 69350 \\
\hline
\end{tabular}

Fonte: DATASUS (2013-2017)

\section{Piscussão}

Avaliar a morbimortalidade infantil nos remete a observar fatores relacionados eventos de morte e adoecimento, uma vez que no Brasil, embora ao longo das adas note-se importante decréscimo ainda há altos índices, mortes e internações pitalares evitáveis a partir do acesso aos serviços de saúde em tempo oportuno, 


\section{ciênciála
pural}

qualidade da assistência ofertada durante o pré-natal, puerpério, e durante as consultas de puericultura.

Estudo realizado em Florianópolis-SC com o objetivo de analisar os fatores de risco para o óbito neonatal, observou um total de 86 mortes neonatais dentre uma amostra de 15879 crianças, sendo a taxa de mortalidade de 5,4 por mil nascidos vivos9. Os autores Yifru Berhan e Asres Berhan ${ }^{10}$ destacam ao avaliar a mortalidade perinatal, que para além dos óbitos fetais considera também óbitos ocorridos até o $7^{\circ}$ dia de vida, diversos parâmetros dentro da assistência de saúde e existem alguns fatores importantes que contribuem com esse indicador, tais como as consultas de pré-natal, a paridade, idade gestacional, prematuridade, peso ao nascer, sexo fetal e a qualidade da assistência no parto.

Um outro estudo realizou uma investigação observando que entre os anos de 1996 até 2015 mostraram um total de 553.718 mortes no Brasil, a tendência desses óbitos também foram analisados com base nos capítulos do CID-10, assim como o estudo, sendo um desses capítulos o XVI. Assim como na pesquisa, o artigo revela que o Sudeste e o Nordeste são os estados que apresentaram os maiores números, com 218.858 e 175.591 mortes, respectivamente ${ }^{11}$.

Observa-se ainda informações ${ }^{12}$ semelhantes às apresentadas, sendo um artigo realizado com bebês de baixo peso após a alta hospitalar, nele foi possível ver que de uma amostra de 190 crianças, 32 bebês vieram a óbito, deles 16 foram por sepse, 10 foram hospitalizados e faleceram lá, 2 morreram a caminho do hospital e os outros 4 mostraram sinais de indisposição. Fica claro então que a prematuridade faz com que o lactente fique vulnerável predispondo-o a entrar nos índices de morbimortalidade.

O estudo realizado por Kokeb ${ }^{13}$ traz aspectos contribuintes comuns nas mortes neonatais, foram principalmente a prematuridade, sepse neonatal, asfixia perinatal, neonatal e aponta que em países em desenvolvimento a causa prevalente são as infecções. Já Santos ${ }^{14}$ refere malformações, causas externas, algumas doenças infectoarasitárias e enfermidades perinatais como razão de óbitos no período pós-neonatal.

Nesse contexto, Sousa ${ }^{15}$ também estudou sobre fatores responsáveis na tribuição dos índices de morbidade. Esse estudo foi elaborado no estado de 
Sergipe, com uma população de 154 recém-nascidos e avaliou a preponderância da morbidade de acordo com diferentes fatores e sistemas e as que mais contribuíram para as internações hospitalares foram: no sistema respiratório, a presença da membrana hialina 157 (99,4\%), no sistema cardíaco. A prevalência foi de casos com agravo no canal arterial patente (PDA), com 62 (39,2\%) ocorrências e no neurológico temos a hemorragia do sistema nervoso central com $27(17,1 \%)$ casos.

O trabalho desenvolvido por Sobieray ${ }^{16}$ se assemelha ao presente estudo, no que diz respeito a também ter analisado mortes perinatais em diferentes anos. Ele observou números consideráveis no município de Curitiba, no ano de 2002, no qual ocorreram 314 óbitos perinatais, em 2003 foram 322 mortes, seguidos de 284 mortes no ano de 2004, e por fim 293 casos no ano de 2005.

A mortalidade e morbidade infantil ainda apresenta altos índices no Brasil principalmente na região Nordeste. Oliveira ${ }^{17}$ cuja pesquisa foi feita por meio das fichas de óbitos de 2011 a 2013 descreve que em Recife ocorreram 843 mortes, sendo o coeficiente de mortalidade infantil de 12,4 para cada 1000 nascidos vivos.

Outra pesquisa feita no nordeste brasileiro, mas especificamente na Bahia, a respeito da mortalidade, demonstra que em grande parte os casos são evitáveis. A proporção de mortes evitáveis no período estudado (2000 à 2012) manteve-se com os maiores valores, ele afirma também que a taxa de mortalidade infantil variou de 26,63 para 17,01 por 1000 nascidos vivos ${ }^{18}$.

Fundamentando os achados temos os conhecimentos formulados por Rocha ${ }^{19}$ que traz um artigo feito no estado do Ceará na qual avaliaram os triênios 2000-2002 e 2010-2012 e observaram 397.200 hospitalizações de crianças menores de cinco anos, dessas 176.545 eram menores de um ano, essa também foi a faixa etária que apresentou maior redução das taxas $(44,8 \%)$ dentro dos anos estudados. Percebe-se que o grupo das gastroenterites foi o que apresentou maior quantidade de internações 33.371 $3,12 \%)$ seguido da pneumonia bacteriana 10.388 (16,54\%) e da asma 8.350 (13,29\%). ota-se então que semelhante ao encontrado na pesquisa, o artigo também traz tipos transtornos respiratórios como sendo os principais responsáveis pelas internações. 


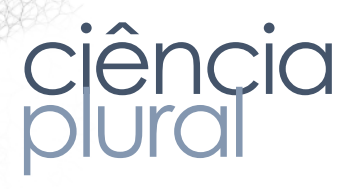

A Atenção Integrada às Doenças Prevalentes na Infância (AIDPI) foi criada com a finalidade de reduzir os índices de morbidade e mortalidade infantil. Isso é feito por meio da melhora da qualidade do atendimento prestado por profissionais de saúde, especialmente nos serviços de atenção básica. Dentre as doenças que a AIDPI procura combater estão os problemas gastrointestinais como a diarreia, os respiratórios como a pneumonia, as infecções, dentre diversos outros ${ }^{20}$.

Por fim, há um estudo que também avalia casos de morbidade infantil onde foi verificada a morbidade no ano de 2010 até 2013 na qual ocorreram 498.466 internações hospitalares de crianças. Dentro desse público foi possível ainda notar que 7525 foram de internações pediátricas em hospitais públicos pós-neonatais, alguns deles com quadro mais grave chegando a ficar em Unidades de Terapia Intensiva (UTI), dos pacientes que tinham entre 1 e 12 meses de vida, 2292 deles ficaram em UTI especializadas e 42 em UTI geral ${ }^{21}$.

Tem-se que os avanços significativos da redução da taxa de mortalidade infantil nas últimas décadas decorreram da efetivação de políticas que tinha como objetivo foralecer a saúde pública devido aos Objetivos de Desenvolvimento do Milênio estabelecidos pela Organização das Nações Unidas. Através da aplicação dessas políticas é possível mapear as evidências que levam a mortalidade infantil e delinear novas estratégias para continuar a prosseguir na redução desses números ${ }^{22}$.

Diante dos estudos realizados na última década é possível afirmar que os óbitos que ocorrem na população infantil na sua grande maioria são devido a causas evitáveis ou tratáveis. Källander ${ }^{23}$ reforçam ainda que os genitores por motivos de não reconhecer os sinais de agravo da enfermidade acabam não levando as crianças até a atenção primária para avaliar ou tratar a doença, do mesmo modo acontece de alguns pais levarem a criança quando o quadro já não pode ser assistido pela atenção básica.

\section{onclusões}

Observou-se nesta pesquisa a morbimortalidade em crianças menores de um relacionado aos agravos do período perinatal; destacando ainda entre os estados 
da região Nordeste alguns com maiores prevalências. Tal fato, sinaliza a necessidade da realização de pesquisas para avaliar as condições de saúde local, os fatores envolvidos, bem como a realização de estratégias em busca de melhores resultados.

Verificou-se a septicemia como o agravo registrado como causa do maior número de mortes. Além dele, houve destaque também para o de outras afecções maternas. Quanto a morbidade hospitalar, os agravos mais prevalentes foram os transtornos respiratórios do período perinatal e as afecções do período perinatal. Dessa forma, as causas perinatais são um dos principais fatores responsáveis por causar mortes e internações hospitalares, tornando-se necessária a tomada de medidas, tais como: incentivo da população a levar as crianças nas consultas de Crescimento e Desenvolvimento, gestantes fazerem o pré-natal, além de recorrer para que haja uma assistência à saúde primária de qualidade e que os índices de procura ao serviço sejam aumentados.

\section{Referências}

1- Maia LTS, Souza WV, Mendes ACG. Determinantes individuais e contextuais associados à mortalidade infantil nas capitais brasileiras: uma abordagem multinível. Cad. Saúde Pública. Rio de Janeiro. 2020; 36 (2). Disponível em: <https://doi.org/10.1590/0102-311X00057519>. ISSN 1678-4464. Acesso em: 04/06/2020.

2- Ramalho AA, Andrade AM, Martins FA, Koifman RJ. Tendência da mortalidade infantil no município de Rio Branco, AC, 1999 a 2015. Rev. Saúde Pública, São Paulo. 2018; 52 (33): 1-11. Disponível em: http://www.scielo.br/pdf/rsp/v52/pt_0034-8910-rsp-52-33.pdf. Acesso em: 20/07/2019.

3- Liu L, Oza S, Hogan D, Perin J, Rudan I, Lawn JE, et al. Global, regional, and national causes of child mortality in 2000-13, with projections to inform post2015 priorities: an updated systematic analysis. The Lancet. 2015; 385:430-40. Disponível em: https://doi.org/10.1016/S0140-6736(14)61698-6. Acesso em: 20/07/2019.

4- Cerda J, Bambs C, Vera C. Infant morbidity and mortality attributable to prenatal smoking in Chile. Rev Panam Salud Publica. 2017; 41: 1-7. Disponível em: https://www.scielosp.org/pdf/rpsp/2017.v41/e106/en. Acesso em: 20/07/2019.

5- Puranik S, Forno E, Bush A, Celedón JC. Predicting Severe Asthma Exacerbations in Children. American Journal of Respiratory and Critical Care Medicine. 2017; 195: 1-6. Disponível em: 
https://www.atsjournals.org/doi/full/10.1164/rccm.201606-

1213PP\#readcube-epdf. Acesso em: 20/07/2019 às 22h24min.

6- Moura EC, Moreira MCN, Menezes LA, Ferreira IA, Gomes R. Complex chronic conditions in children and adolescents: hospitalizations in Brazil, 2013. Ciência \& Saúde Coletiva. 2017; 22(8): 2727-2734. Disponível em: http://dx.doi.org/10.1590/1413-81232017228.01992016. Acesso em: 20/07/2019.

7- Bando DH, Lester D, Machado MFS, Pires ESMR, Barrozo LV. Estudo ecológico sobre suicídio e homicídio no estado de minas gerais, brasil. Rev Espaço e Geografia. 2015; 18: 397:417. Disponível em: http://lsie.unb.br/espacoegeografia/index.php/espacoegeografia/article/vi ew/446. Acesso em 25/07/2019.

8- Instituto Brasileiro de Geografia e Estatística (IBGE). 2019. Disponível em: https://www.ibge.gov.br/cidades-e-estados.html?view=municipio. Acessado em: 04/11/2019.

9- Garcia LP, Fernandes CM, Traebert J. Risk factors for neonatal death in the capital city with the lowest infant mortality rate in Brazil. J. Pediatr. (Rio J.). 2019; 95 (2): 194-200. Disponível em: http://dx.doi.org/10.1016/j.jped.2017.12.007. Acesso em: 05/08/2019.

10-Berhan Y, Berhan A. A meta-analysis of selected maternal and fetal factors for perinatal mortality. Ethiop J Health Sci. 2014; 24: 55-68. Disponível em: https://www.ncbi.nlm.nih.gov/pmc/articles/PMC4249209/pdf/EJHS240S0055.pdf. Acesso em: 31/10/2019.

11- Barros PS, Aquino EC, Souza MR. Mortalidade fetal e os desafios para a atenção à saúde da mulher no Brasil. Rev Saúde Pública. 2019; 53: 1-10. Disponível em: https://doi.org/10.11606/S1518-8787.2019053000714. Acesso em: 05/08/2019.

12- Abdallah Y, Namiiro F, Nankunda J, Magalu J, Vaucher Y. Mortality among very low birth weight infants after hospital discharge in a low resource setting. BMC Pediatrics. 2018; 18:239. Disponível em: https://doi.org/10.1186/s12887018-1226-4. Acesso em: 18/10/2019.

13-Kokeb M, Desta T. Institution Based Prospective Cross-Sectional Study on Patterns of Neonatal Morbidity at Gondar University Hospital Neonatal Unit, North-West Ethiopia. Ethiop J Health Sci. 2016; 26 (1): 73-79. Disponível em: https://www.ncbi.nlm.nih.gov/pmc/articles/PMC4762962/. Acesso em: 18/10/2019.

14-Santos EP, Ferrari RAP, Bertolozz MR, Cardelli AAM, Godoy CB, Genovesi FF. Mortalidade entre menores de um ano: análise dos casos após alta das maternidades. Rev Esc Enferm USP. 2016; 50(3): 390-398. Disponível em: http://dx.doi.org/10.1590/S0080-623420160000400003. Acesso em: 22/07/2019.

15- Sousa DS, Sousa Júnior AS, Santos ADR, Melo EV, Lima SO, Almeida Santos MA, et al. Morbidade em recém- nascidos prematuros de extremo baixo peso em unidade de terapia intensiva neonatal. Rev. Bras. Saúde Matern. Infant. 
2017; 17(1): 149-157. Disponível em: http://dx.doi.org/10.1590/180693042017000100008.Acesso em 21 de julho de 2019

16-Sobieray NLEC, Urbanetz AA, Tristão EG. Estudo da mortalidade perinatal do município de Curitiba no período de 2002 a 2005. Arq Med Hosp Fac Cienc Med Santa Casa São Paulo. 2015; 60: 47-53. Disponível em: http://arquivosmedicos.fcmsantacasasp.edu.br/index.php/AMSCSP/article /view/149/155. Acesso em: 06/11/2019

17- Oliveira CM, Guimarães MJB, Bonfim CV, Frias PG, Antonino VCS, Guimarães ALS, et al. Adequação da investigação dos óbitos infantis no Recife, Pernambuco, Brasil. Ciênc. saúde coletiva. 2018; 23 (3): 701-714. Disponível em: http://dx.doi.org/10.1590/1413-81232018233.14052016. Acesso em: 06/11/2019.

18- Tavares LT, Albergaria TFS, Guimarães MAP, Pedreira RBS, Pinto Junior EP. Mortalidade infantil por causas evitáveis na Bahia, 2000-2012. Rev Eletron Comun Inf Inov Saúde. 2016; 10(3): 1-10. Disponível em: https:/ / www.reciis.icict.fiocruz.br/index.php/reciis/article/view/1044/pdf 1044. Acesso em: 10/11/2019.

19- Rocha DM, Costa LQ. Internações evitáveis na Atenção Primária em menores de cinco anos na macrorregião de saúde de um estado do nordeste: comparação entre os triênios 2000-02 e 2010-12. Actas de saúde colet. 2017; 11(4): 91-104. Disponível em: http://dx.doi.org/10.18569/tempus.v11i4.1980. Acesso em: 06/11/2019.

20- Ministério da saúde. Organização Pan-Americana da saúde. Fundo das nações unidas para a infância. Manual Aidpi criança - 2 meses a 5 anos. Brasília - DF. 2017. [Acesso em: 20/08/2019]. Disponível em: http:/ / portalarquivos.saude.gov.br/images/pdf/2017/julho/12/17-0056Online.pdf.

21- Ibiebele I, Algert CS, Bowen JR, Roberts CL. Pediatric admissions that include intensive care: a population-based study. BMC Health Services Research. 2018; 18: 264. Disponível em: https://doi.org/10.1186/s12913-018-3041-x. Acesso em: 07/11/2019.

22-Deribew A, Tessema GA, Deribe K, Melaku YA, Lakew Y, Amare AT, et al. Trends, causes, and risk factors of mortality among children under 5 in Ethiopia, 1990-2013: findings from the Global Burden of Disease Study 2013. Population health metrics. 2016; 14: 42. Disponível em: 10.1186/s12963-0160112-2. Acesso em: 10/11/2019.

23-Källander K, Counihan H, Cerveau T, Mbofana F. Barriers on the pathway to survival for children dying from treatable illnesses in Inhambane province, Mozambique. Journal of global health. 2019; 9 (1): 1-14. Disponível em: 10.7189/jogh.09.010809. Acesso em: 15/09/2019. 